\title{
Neural network monitoring of resistance welding processes
}

\author{
J.M. Quero, R.L. Millan, L.G. Franquelo and J. Cañas \\ Escuela Superior de Ingenieros , Universidad de Sevilla
}

\begin{abstract}
Summary: Control of weld quality is one of the most important and complex processes to be carried out on production lines. Neural Networks have shown good results in fields such as modelling and control of physical processes. It is suggested in this article that a neural classifier should be used to carry out non-destructive on-line analysis. This system has been developed and installed at resistance welding stations. Results confirm the validity of neural networks used for this type of application.
\end{abstract}

\section{Introduction}

The resistance welding methods used for numerous industrial applications where high-output production is required have reached a high degree of reliability. One special case in point is the automobile industry where the requirements of an increasingly competitive market have made it necessary to use high-productivity welding techniques, and to find on-line systems which detect those workpieces in which the joint produced is defective.

Various non-destructive on-line control techniques have been developed in order to establish the quality of the welded joint obtained. A control system generally comprises three basic elements: a transmitter, a receiver and a discriminator. The transmitter is a transducer which excites the welded workpiece, whose response to excitation is picked up in another transducer which in turn passes on the signal to a device which decides whether or not the workpiece is to be rejected. Among the various methods of energy excitation ${ }^{1}$ special mention should be made of ultrasonics, X-rays and electromagnetic waves.

Major drawbacks of the techniques mentioned are high equipment cost, lack of flexibility in their application and the time required to test the weld, all of which means that application to a production line is almost unviable.

The topic of modelling the physical process of welding based on on-line control has been widely studied ${ }^{2}$ with the objective of relating welding control parameters to the mechanical properties which would be obtained from previous tests. This methodology does not, however, guarantee that the weld produced will meet expectations because of the existence of a combination of uncontrolled parameters which may affect joint quality, such as impurities in the surfaces to be joined, the state of these surfaces, etc.
In the present work, following the line taken in previous investigations, a supervision system has been developed which makes it possible to predict on-line the ultimate strength of the joint as a function of welding parameters obtained directly from the process. If the calculated ultimate strength is below a certain threshold the workpiece is rejected. The supervision system set-up uses neural networks, application of which has been successfully developed in fields such as recognition of patterns or control of industrial processes. ${ }^{3}$ Such networks are proposed as classifiers to determine the soundness of the weld.

The welding process used is described hereunder with special reference to control parameters. The relationship between the values of the physical quantities measured and linked together in the process and the ultimate strength obtained experimentally are studied. Then, the paper presents the neural networks, describing them with special attention to their application as classifiers. Finally, the paper describes the application developed, analysing the data obtained on the production line.

\section{Description of welding station}

From this point onwards, the term resistance welding station ${ }^{4}$ will be used. It should be pointed out, however, that the supervision system proposed can easily be applied to other types of station.

At the stations considered, a shaft/socket assembly is joined by means of a pure resistance welding process, as shown in Fig. 1. This welding station has a controller ${ }^{4}$ which governs the current source which, in this case, is a rectifier.

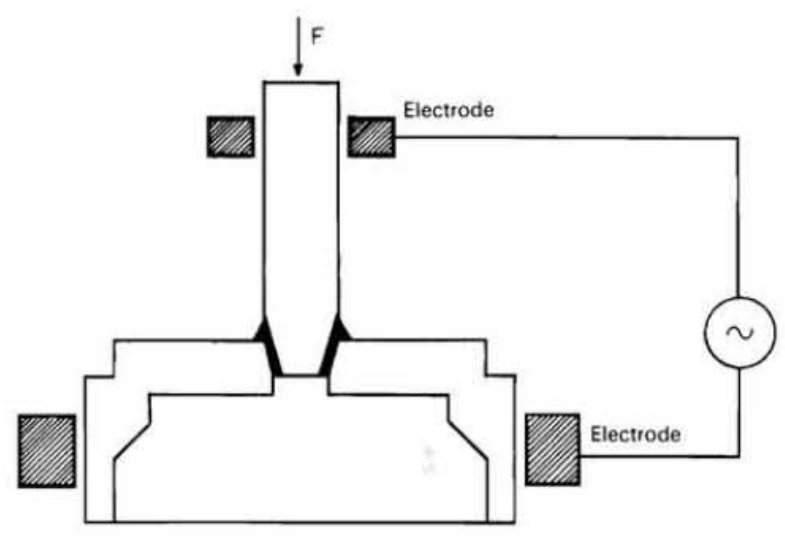

1 Joint cross-section. 


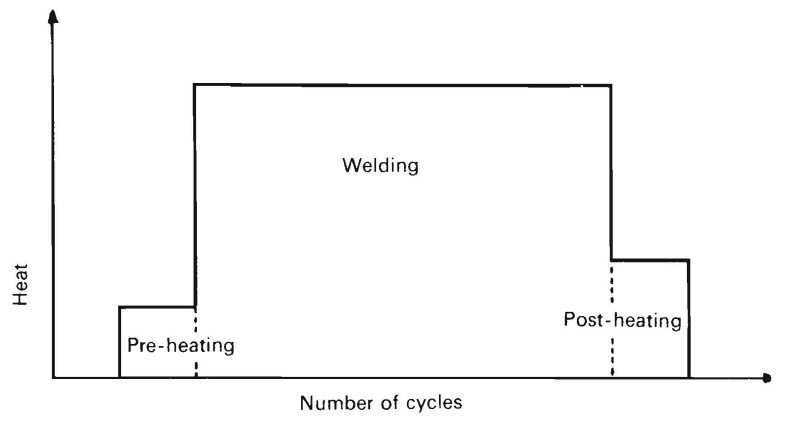

2 Heat input to joint during welding.

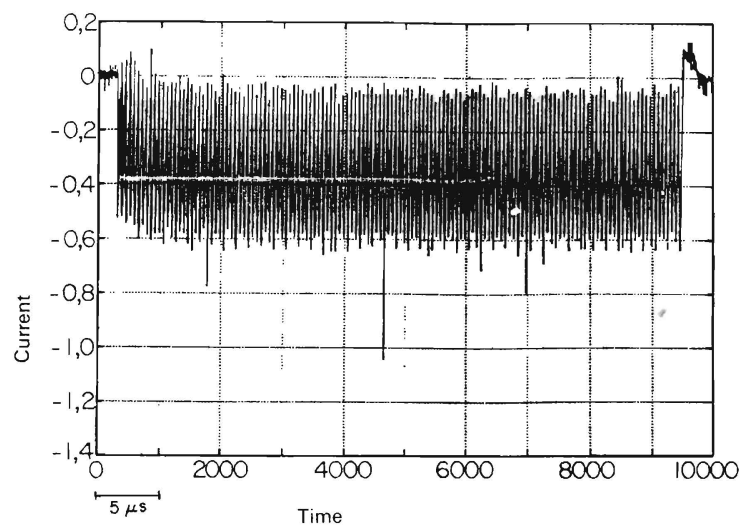

3 Waveform for a specimen with an ultimate strength of $3660 \mathrm{~K}$. Sampling period $50 \mu \mathrm{sec}$.

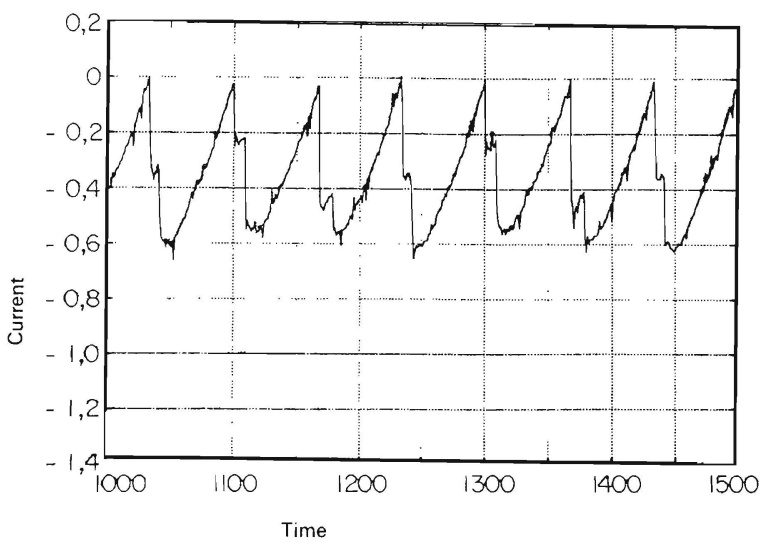

4 Detail of previous figure.

The energy supplied to the joint is determined as a function of the phase angle of the rectifier in each cycle, the sinusoidal waveform of the supply voltage, and the number of complete cycles which constitute welding time.

The welding phases are: pre-heating, heating and post-heating (Fig. 2) corresponding to the number of pre-heating cycles, the number of welding cycles and the number of cooling cycles respectively. In each phase the triggering angle of the rectifier is defined as a percentage but, in the satisfactory development of a weld, in addition to the geometric and energy parameters already explained, there are other relevant uncontrolled factors such as metallurgical factors (impurities on the surface, oxides and surface treatments such as chromium plating), ambient contamination, etc. All these factors affect the ultimate strength finally obtained in the joint, and their presence establishes the need for a system to supervise weld quality.

Because of the nature of the welding operation, the
Table 1 Ultimate strength values, $\mathrm{kg}$

\begin{tabular}{lllll} 
Order & Normal & Oxidised & $\begin{array}{l}\text { Chromium- } \\
\text { plated }\end{array}$ & $\begin{array}{l}\text { Chromium- } \\
\text { plated }\end{array}$ \\
\hline 1 & 5090 & 3886 & 3400 & 4500 \\
2 & 4802 & 4402 & 3902 & 4850 \\
3 & 5190 & 3872 & 4386 & 3966 \\
4 & 5600 & 3786 & 4516 & 5010 \\
5 & 4884 & 2810 & 3810 & 4896 \\
6 & 4862 & 3358 & 4384 & 3792 \\
7 & 5022 & 2390 & 2900 & 4138 \\
8 & 4720 & 3610 & 4270 & 4026 \\
9 & 5080 & 2450 & 4098 & 4672 \\
\hline
\end{tabular}

Table 2 Current form factors

\begin{tabular}{lllll}
\hline & & & & \\
Order & Normal & Oxidised & plated & $\begin{array}{l}\text { Chromium- } \\
\text { plated }\end{array}$ \\
\hline 1 & 0.3574 & 0.0122 & 0.2211 & 0.2960 \\
2 & 0.4587 & 0.2283 & 0.2198 & 0.2977 \\
3 & 0.5629 & 0.1170 & 0.3240 & 0.2877 \\
4 & 0.4723 & 0.1348 & 0.3148 & 0.3133 \\
5 & 0.4430 & 0.1140 & 0.2939 & 0.3586 \\
6 & 0.4590 & 0.0830 & 0.2531 & 0.3980 \\
7 & 0.4102 & 0.0168 & 0.1995 & 0.3176 \\
8 & 0.3857 & 0.0000 & 0.3015 & 0.3068 \\
9 & 0.3980 & 0.0456 & 0.3372 & 0.2954 \\
\hline
\end{tabular}

recording of the electrical current waveform passing through the zone where the weld is formed contains the history of the joint. As shown in Fig. 3 and 4, however, classification based on the waveforms generated requires a detailed study of their forms.

\section{Relationship between ultimate strength and current curves}

In order to study the relationship between the waveforms recorded and the corresponding ultimate strength values, 36 specimens were tested which were divided into four groups characterised by their surface state. Table 1 shows the ultimate strength values obtained from tensile tests.

Based on the current waveform data recorded by oscilloscope, the form factor for each wave was calculated, being defined as:

$I_{f f i}=\frac{\sqrt{\sum_{k=i}^{N} i^{2}}}{N}$

where $i$ is the sampled value of the current circulating between the electrodes of the welder and $N$ is the number of samples. The values obtained for the specimens in Table 1 are shown in Table 2, normalised from 0 to 1 .

Statistical analysis of Tables 1 and 2 based on Student's $T$ confirms the existence of a correlation greater than $95 \%$ between the ultimate strength and the $I_{\mathrm{ffi}}$ value, which validates the hypothesis that a function exists by which these are related.

Figure 5 is a graph showing the relationship between the ultimate strength and $I_{\mathrm{ffi}}$. The linear correlation 


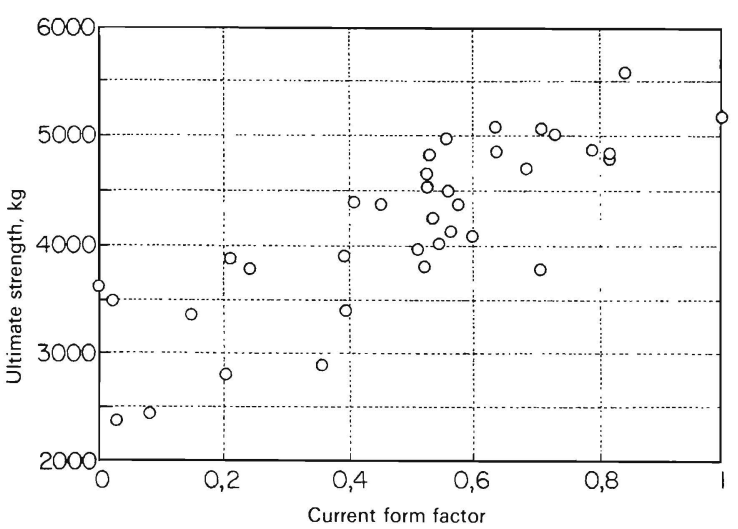

5 Ultimate strength against current form factor.

coefficient between the two variables is low (0.63), indicating a need to approximate by means of functions more complex than the linear, using a larger volume of information and more powerful classifiers.

In order to increase the volume of information considered it was decided that a discrete histogram should be used, dividing the sampled current intensity curve into eight energy levels. One of the advantages of using this histogram is that it is easy to obtain by simple digital electronics, allowing it to be calculated at low computing cost or, which is the same thing, in real time. Table 3 shows the histograms obtained for the specimens listed in Table 1.

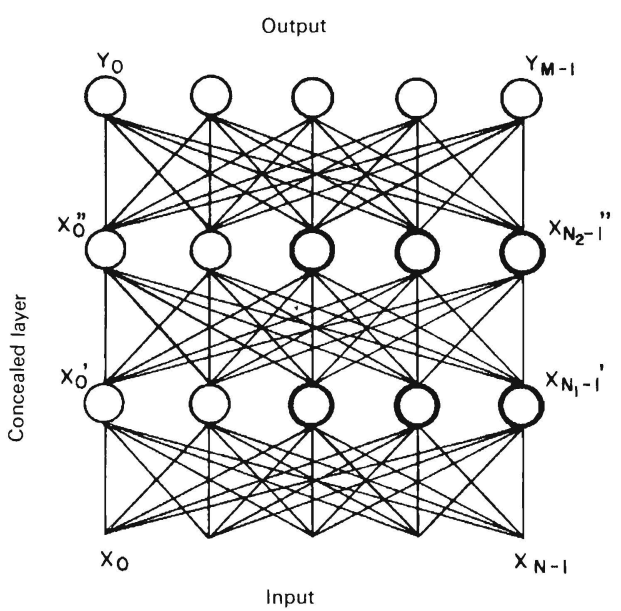

6 Network for NDT pattern classification.

\section{Neural networks}

Multi-layer perception has been successfully used for identification and classification of patterns in non-destructive tests. These networks consist of various layers of closely inter-connected units operating in parallel, as shown in Fig. 6.

Each unit generates an internal activity proportional to the weighted sum of its input signals. Its output is produced by applying the function $\gamma$ (shown in Fig. 7) to the internal activity.

Table 3 Test histograms

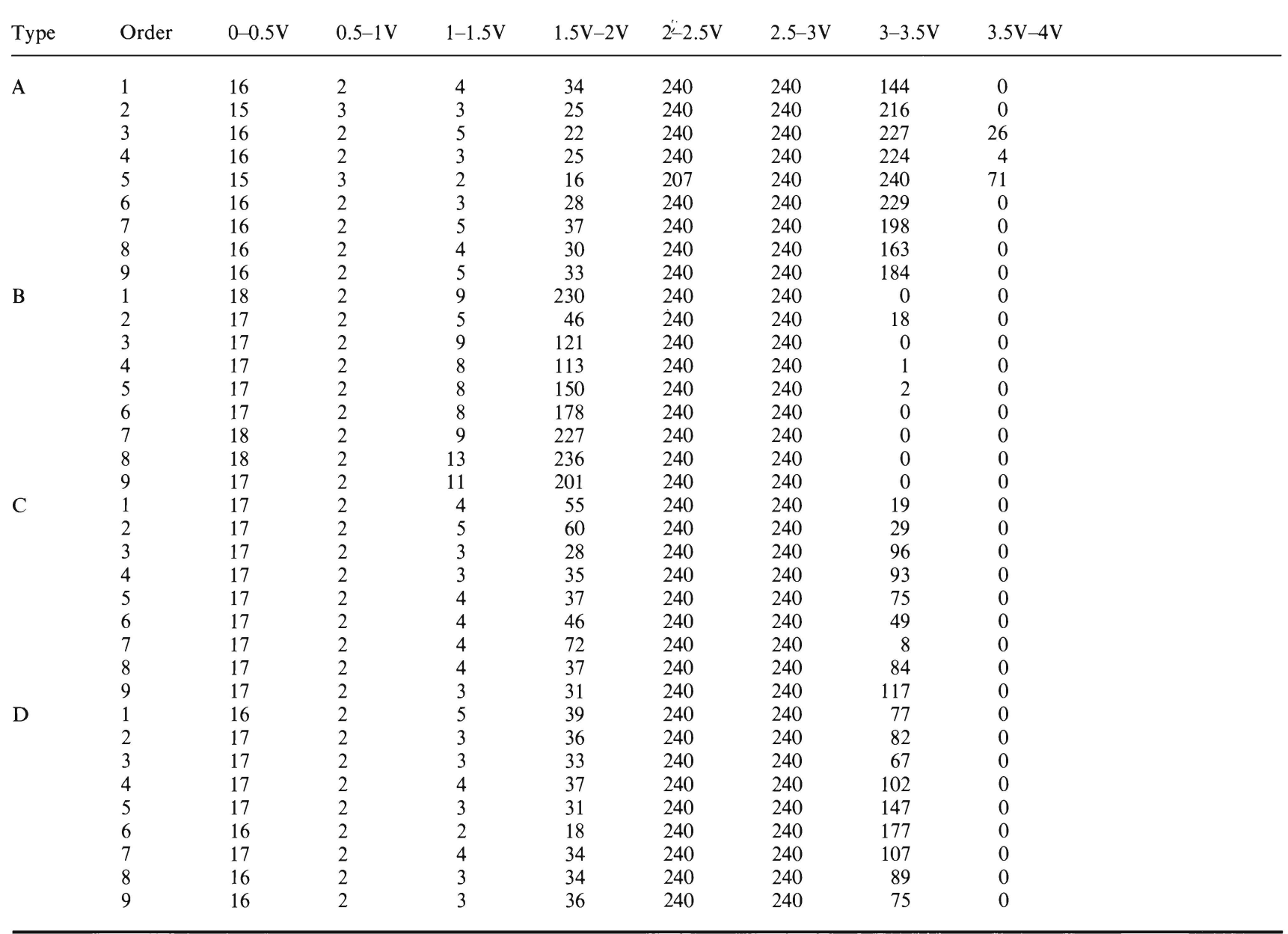




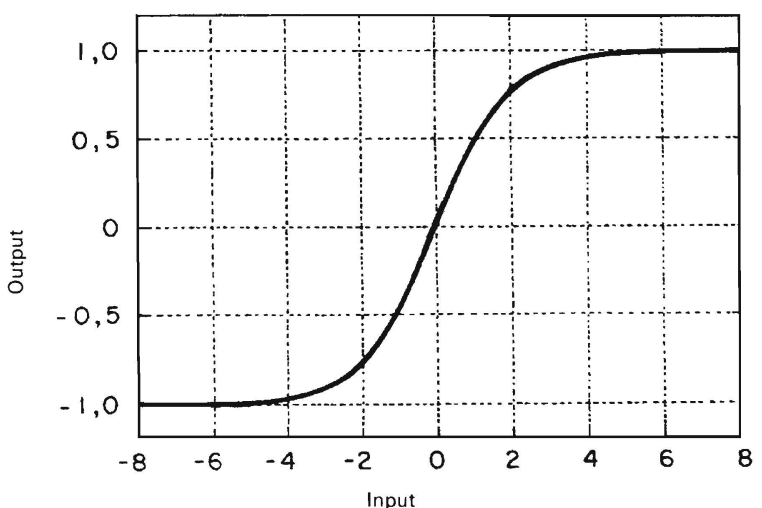

7 Output function $\gamma(\cdot)$ of a neuron.

$$
\mathrm{O}_{\mathrm{i}}=\gamma\left(\sum_{\mathrm{i}=0}^{\mathrm{N}} \omega_{\mathrm{ij}} \mathrm{l}_{\mathrm{j}}\right)
$$

The back-propagation learning algorithm ${ }^{5}$ is used to obtain a desired group of connections approximating to a given function. The weights of each connection $\omega_{\mathrm{ij}}$ are modified in an amount proportional to the product of an error signal $\delta_{\mathrm{j}}$ linked to the said connection by the output from the unit to which it belongs, $o_{\mathrm{i}}$,

$$
\Delta_{\mathrm{p}} \omega_{\mathrm{ij}}=\eta \delta_{\mathrm{pj}} \mathrm{O}_{\mathrm{pi}}
$$

where $p$ represents the index of the pattern on which the learning process is particularised.

Determination of the error signal is a recurring process which begins in the output units:

$$
\delta_{p j}=\left(t_{p j}-o_{p j}\right) \gamma_{j}^{\prime}\left(\sum_{i} \omega_{p i} o_{p i}\right)
$$

where $t_{\mathrm{pj}}$ is the jth component of desired output when the input pattern $p$ is applied to the network and $\gamma($.$) is the$ derivate of the neuron transfer function. The signal error for the concealed units, for which there is no known desired output, is defined as:

$$
\delta_{\mathrm{pj}}=\gamma_{\mathrm{j}}^{\prime}\left(\sum_{\mathrm{i}} \omega_{\mathrm{pi}} \mathrm{O}_{\mathrm{pi}}\right) \sum_{\mathbf{k}} \delta_{\mathrm{pk}} \omega_{\mathbf{k j}}
$$

This algorithm is applied until the network, for each input applied, generates the desired output with a maximum error.

\section{Application}

To establish the validity of the method formulated a weld monitoring system was designed for which a block diagram is shown in Fig. 8.

For this application a specific data acquisition system was developed, of which a working diagram is given in Fig. 9. This system comprises eight counters which show increments as a function of the analogue/digital

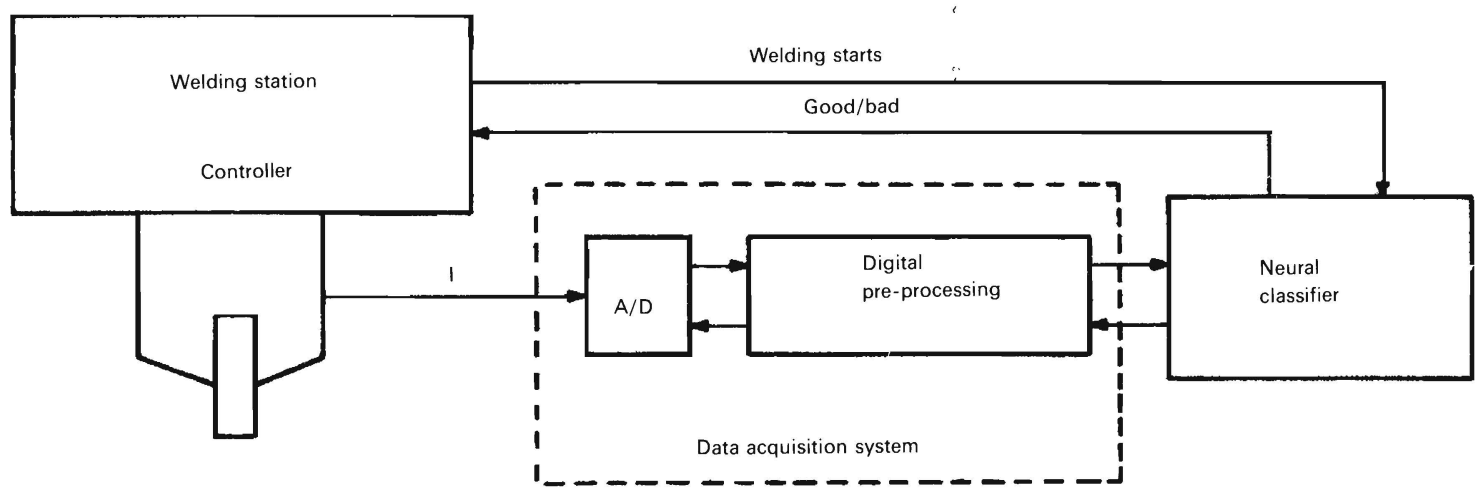

8 Block diagram of weld monitoring system.

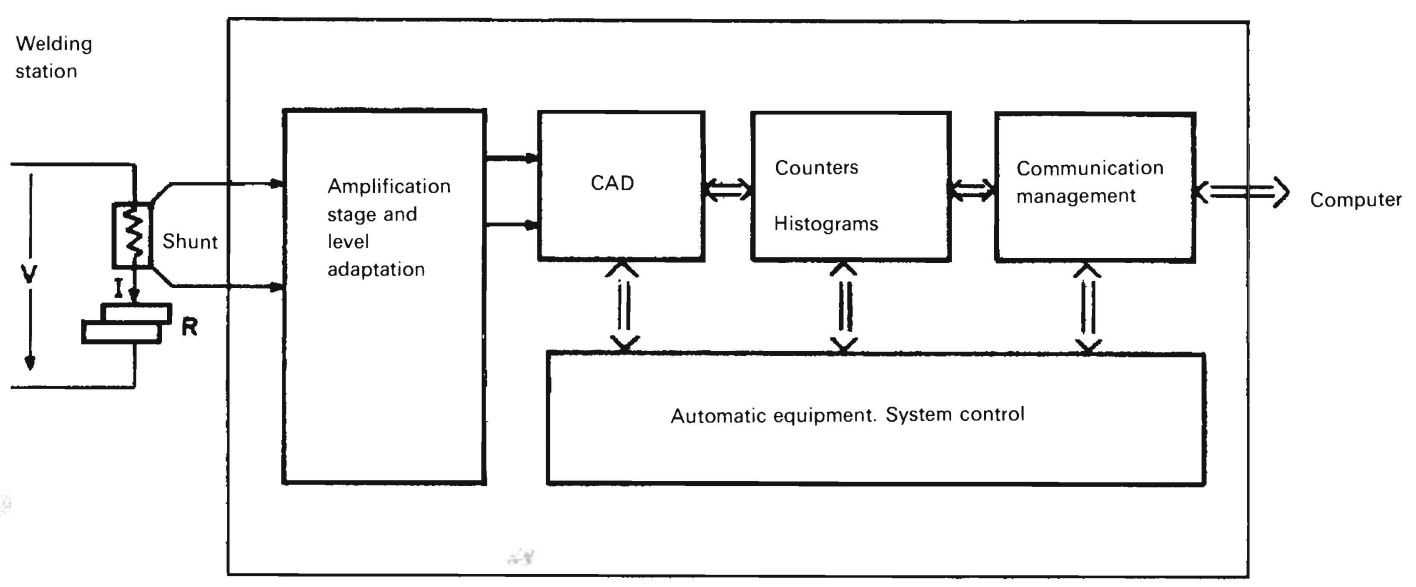


conversion of the current signal. The signal is sampled every $50 \mu \mathrm{sec}$ throughout the welding process $(0.5 \mathrm{sec})$. This specific hardware makes it possible to calculate the histogram, i.e. the input data for the neural network, as soon as welding finishes. These data are read by the control computer and the neural network located in the computer memory is activated. For this purpose the neural network was previously configured in a learning phase using the data given in Table 3. The neural network selected has a concealed layer with 25 units and an output neuron. The number of iterations required in the learning process, which is carried out at a SUN 10 workstation, was 450000.

The system was checked by installing it on the production line. The total number of specimens tested was 450 . Classification results were: $98 \%$ of samples correctly classified (438 correctly welded and three with an ultimate strength less than what was required) while the remaining $2 \%$ generated intermediate output in the neural network or which is the same thing, classification in a zone of uncertainty coinciding with ultimate strength values very close to the minimum permissible.

\section{Conclusions}

This paper describes a weld supervision system based on neural networks. The system represents an initial phase in learning to classify welds produced on the basis of data obtained as they are formed. In a second phase, the system was installed on a production line allowing real-time quality control of welding. The system was checked on 450 specimens, all of which were correctly classified. The same technique is now being applied to gas-shielded electric arc welding (MIG/MAG) using sliding windows. ${ }^{6}$

\section{References}

[1] Hull B and John V: 'Non-destructive testing'. MacMillan Education, London, 1988.

[2] Andersen K, Cook G E, Karsai G and Ramaswamy K: IEEE Trans Ind App/ 199026 (5).

[3] Quero J M, Franquelo L G and Camacho E F. 'Neural network for constrained predictive control' IEEE Trans Circuits and Systems 1993 vol. 40 issue 9, pp. 621-626.

[4] 'Weld quality monitor instruction manual'. SRL Controls Division, 1981.

[5] Rumelhart D and McClelland J: 'Parallel distributed processing'. Publ MIT Press, 1986.

6 Anderson J A and Rosenfeld E: 'Neurocomputing'. Publ MIT Press, 1988. 